\section{Effect of aversive stimulation and early handling on skin conductance defecation, and activity in Mus musculus*}

\author{
ROBERT DEUTSCH and LARRY E. ROBERTS \\ McMaster University, Hamilton, Ont., Canada
}

Presentation of an aversive stimulus (sight of $\mathrm{E}$ ) always elicited an increase in skin conductance and defecation, but the locomotor response was a decrease early in testing and an increase later on. Early handling abolished each component of the response pattern. These results suggest control of the electrodermal system by a mechanism that regulates emotional defecation and agonistic locomotor responding. Autonomic responses occur whenever this mechanism is aroused, but the locomotor response that follows depends upon cues that are present and S's previous experience with them.

When an aversive stimulus is presented to a mouse, a pattern of responses is observed that includes defecation, freezing behavior, and occasionally urination as well. The responses of the pattern are usually taken to indicate presence of fear or emotional arousal (Lindzey, Winston, \& Manosevitz, 1963; Hunt \& Otis, 1953). The present experiments determined whether or not a change in skin conductance (SC) is a reliable component of this pattern.

EXPERIMENT 1

Experiment 1 examined the relationship of SC responses to emotional defecation and freezing behavior elicited by visual sighting of $\mathrm{E}$. The purpose was to determine if freezing and defecation are accompanied by an increase in SC and to see whether the three responses follow the same or different courses during repeated testing. Method

The Ss were 25 mice, 55-93 days of age, drawn from the $\mathrm{F} 2$ population of a cross between the $C 57 \mathrm{BL} / 6 \mathrm{~J}$ and $\mathrm{A} / \mathrm{J}$ inbred lines. In order to minimize urination, which would have seriously complicated interpretation of the SC measurements (Roberts, 1967a), only female Ss were studied. Ss were undisturbed from weaning at 28 days of age, except for routine cage cleaning every 2 weeks.

The apparatus was a shuttlebox ( $3^{1 / 2} \times 11 \times 6$ in.) with a removable top that incorporated a one-way mirror. The floor consisted of two independent grids, which were supported by electrical contacts to permit automatic recording of activity (crosses from one grid to the other). A 5-microA current was passed through the grid floor to provide a measure of SC (Roberts, 1967a).

\footnotetext{
*This research was supported by a grant from the National Research Council of Canada to the second author.
}

The $S$ was placed in the apparatus for $35 \mathrm{~min}$. At the start of Minutes 6 , $12,18,24$, and $30, E$ opened the lid of the shuttlebox and brought his face to within $10 \mathrm{in}$. of $\mathrm{S} ; 1 \mathrm{~min}$ later, $E$ withdrew and the lid was closed. These 1-min periods will be referred to as stimulus presentations. Ss were tested on each of 4 consecutive days. SC, activity, defecation, and urination were recorded throughout.

Five SC readings were taken at 12-sec intervals for each of the minutes before, during, and after a stimulus presentation. Medians of SC for each minute of the trial. Conductance readings distorted by feces occasionally touching or shorting out the grid were excluded from the analysis. The significance levels reported herein were taken from repeated-measures analyses of variance in which Ss, minutes (before or during), and either trials or days served as variates.

\section{Results}

Figure 1 portrays SC, defecation, and activity before, during, and after each stimulus presentation on the first day of testing. These findings show that presentation of the stimulus led to a marked increase in defecation and SC on all five trials. On Trial 1 , for example, SC increased for 23 of $25 \mathrm{Ss}$ and defecation increased for all 25 . Locomotor activity was depressed in the presence of the stimulus, indicating freezing behavior, but this did not persist beyond the third trial. As expected, the incidence of urination was very low (Roberts, 1967a). Six Ss urinated once in the presence of the stimulus on the first day of testing, while, on subsequent days, 11 urinations were observed during a total of 400 stimulus presentations.

Figure 2 describes changes in the response pattern as a function of days of testing, averaged over trials. these five values provided a measure of
Presentation of the stimulus elicited a substantial increment in SC and defecation throughout, although the amplitude of these responses diminished slightly as testing proceeded. In contrast to the autonomic responses, which changed relatively little, the direction of the activity response reversed by the fourth day of testing. The reversal was evident within as well as between sessions and was statistically significant in both cases $(p<.01)$. The course and consistency of the reversal are portrayed in greater detail in Fig. 3 , where the amplitude of the activity response (level before the stimulus subtracted from level during) is plotted as a function of trials for each day of testing. Increases in activity were particularly evident on the fourth day, where Ss were observed to move away from $E$ as though attempting to escape.

\section{Discussion}

Figures 1 and 2 show that an increase in SC reliably accompanied emotional defecation and freezing or escape-oriented behavior. This suggests that the electrodermal system of rodents is regulated by a neural mechanism which also controls emotional defecation and agonistic locomotor responding. Recent CER experiments on the mouse (Roberts, 1967b) and rat (Roberts \& Young ${ }^{1}$; Woll, 1968) provide additional support for this conclusion. These studies have

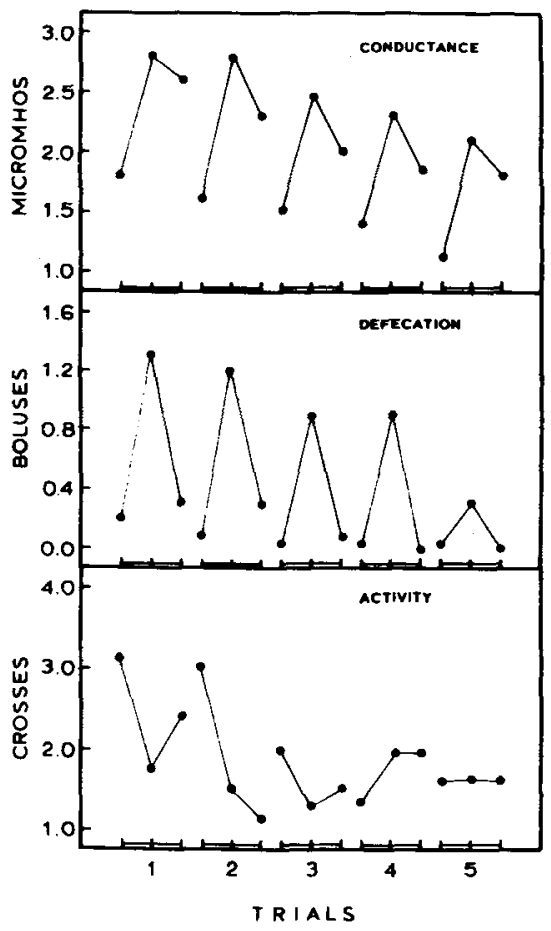

Fig. 1. Effect of stimulus presentation of SC, defecation, and activity on the first day of testing. 


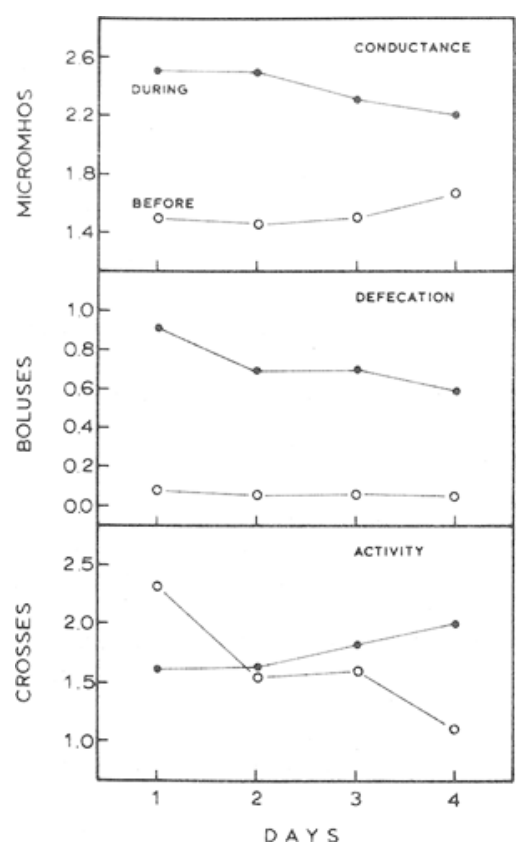

Fig. 2. SC, defecation, and activity before and during stimulus presentations as a function of days of testing.

shown that an increment in electrodermal arousal accompanies the suppression of overt movement and food-reinforced leverpressing and that the electrodermal and suppression responses display similar properties and follow the same course throughout CER acquisition and extinction.

Although freezing behavior was the predominant locomotor response early in testing, increments in activity became more frequent as testing proceeded. This indicates that the locomotor response to $a$ novel and presumably aversive stimulus consists of either freezing or escape-oriented behavior and that degree of prior exposure to the stimulus may be one of the variables that determines which behavior will be observed. Another interesting finding was that the autonomic responses attending the two forms of locomotor response were similar. This suggests that neurons which control electrodermal and eliminatory responses are organized within a mechanism that potentiates, not just one, but several forms of agonistic behavior. The electrodermal and defecation responses occur whenever the mechanism is aroused, but the behavioral response that follows is determined by cues that are present and S's previous experience with them.

An alternative interpretation of the present findings is that the SC response was a by-product of contact area and urination artifacts that might occur when grid techniques of electrodermal measurement are employed (Roberts, 1967a). This view may be rejected because urination occurred on fewer than $5 \%$ of the trials and also because the $\mathrm{SC}$ response was always an increase, even though the locomotor response, a likely determinant of contact area, was an increase, a decrease, or no change at various stages of testing. Nor can an artifact hypothesis account for electrodermal correlates of conditioned suppression; the electrodermal responses reported by Roberts \& Young 1 were recorded by attaching electrodes directly to the feet of $S$, thereby eliminating any possibility of serious measurement error.

\section{EXPERIMENT 2}

If the electrodermal system of the mouse is controlled by a mechanism that also controls emotional defecation and freezing or escape behavior, an experimental manipulation that abolishes eliminatory and locomotor responses to an aversive stimulus should also abolish the SC response. Experiment 2 tested this prediction. The experimental manipulation was early handling, which has been shown to reduce defecation in the open field (Levine, 1962; Levine, Haltmeyer, Karas, \& Denenberg, 1967).



Fig. 3. Activity response (level before subtracted from level during) as a function of trials and days of testing.

Method

The Ss were 12 female mice from the same $\mathrm{F} 2$ population that was the source of $\mathrm{Ss}$ for Experiment 1 . The apparatus was the same as in Experiment 1.

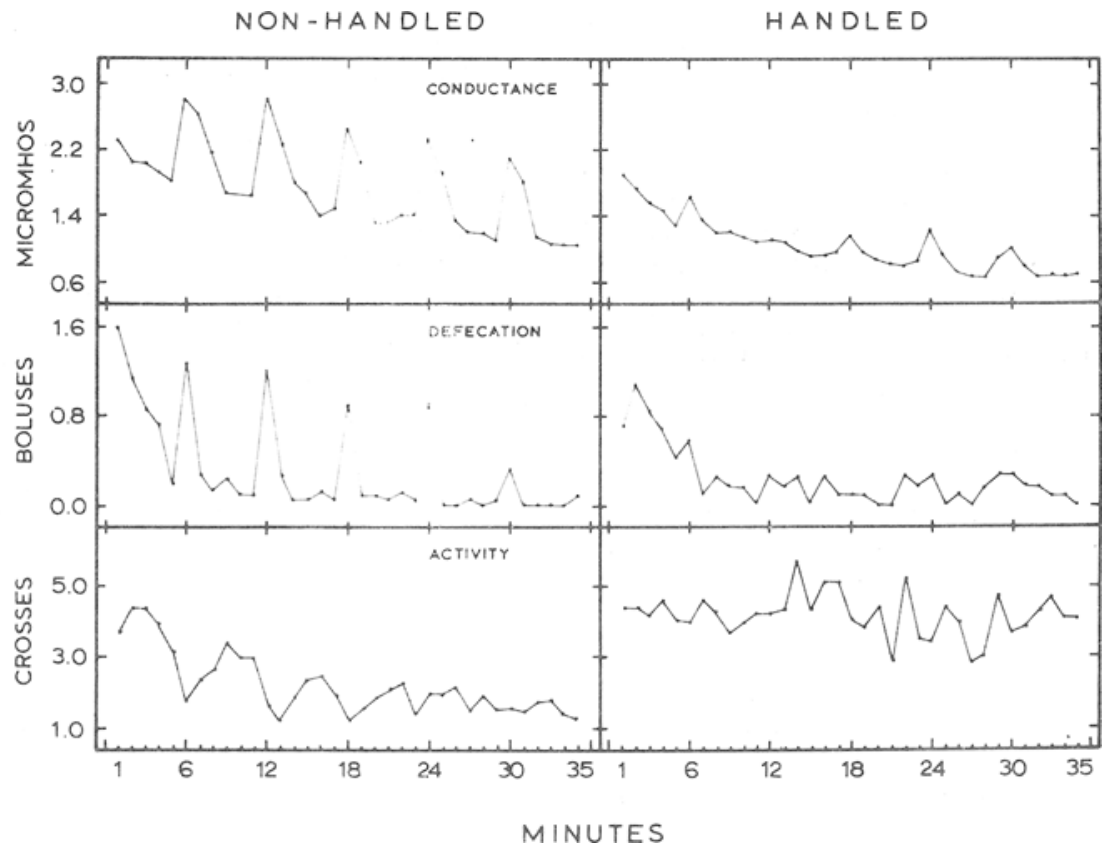

Fig. 4. Minute by minute plot of SC, defecation, and activity for handled and nonhandled Ss. 
The Ss were subjected to daily handling from 3 to 28 days of age. This consisted of removing $\mathrm{S}$ from the home cage, stroking its back for approximately $1 \mathrm{~min}$, and then depositing $S$ on the sawdust floor of a fresh cage. After Ss in each litter had been handled in the same manner, they were returned to the home cage one by one. At 28 days of age, Ss were weaned and thereafter received treatment identical to that given $S s$ in Experiment 1.

The Ss were tested for a single 35-min session at 80 days of age according to the procedure described in Experiment 1.

\section{Results}

Figure 4 compares the behavior of the handled group with the results of the first day of testing in Experiment 1, in which Ss were not exposed to the early handling procedure. Here, SC, defecation, and activity are plotted for each of the 35 min of testing. It is clear that each component of the response pattern was greatly reduced or completely abolished by early handling. Also, the tonic level of activity was much higher in the handled group $(p<.01)$, a finding that is consistent with the results of Levine et al (1967), Denenberg, Schell, Karas, \& Haltmeyer (1966), and others

\section{DISCUSSION}

Experiment 1 showed that a large increment in SC accompanies eliminatory and locomotor responses to an aversive stimulus. Experiment 2 showed that early handling greatly reduces or abolishes the SC, defecation, and freezing responses that normally would have been observed on the first day of testing. These findings provide further evidence for control of the electrodermal system of the mouse by a neural mechanism that regulates emotional defecation and agonistic locomotor responding.

The effect of early handling, which was rather striking, may be interpreted in one of two ways. The first possibility is that early handling lowers susceptibility to emotional arousal (Denenberg, 1964). :A different account, however, attributes the effect of handling to habituation to a specific stimulus situation rather than to a general lowering of emotional reactivity. The possible role of habituation would have been maximized in the present study by the similarity, due to the presence of $E$, of conditions during treatment and testing. The extent to which the effect of early handling is due to habituation, rather than to a lowering of emotional reactivity, is unclear and remains a problem for future research.

\section{REFERENCES}

DENENBERG, V. H. Critical periods, stimulus input, and emotional reactivity: A theory of infantile stimulation. Psychological Review, 1964, 71, 335-351. DENENBERG, V. H., SCHELL, S. F., KAR AS, G. G., \& HALTMEYER, G. C. Comparison of background stimulation and handling as forms of infantile stimulation. Psychological Reports, 1966. 19, 943-948.

HUNT, H. F., \& OTIS, L, Conditioned and unconditioned emotional defecation in the rat. Journal of Comparative \& Physiological Psychology, 1953, 46, 378-382.

LEVINE, S. The effects of infantile experience on adult behavior. In $A$. I Bachrach (Ed.), Experimental foundations of clinical psychology. New York: Basic Books, 1962. Pp. 139-169.

LEVINE, S., HALTMEYER, G. C., KARAS, G. G., \& DENENBERG, V. H. Physiological and behavioral effects of infantile stimulation. Physiology \& Behavior, 1967, 2, 55-59.

LINDZEY, G., WINSTON, H. D., \& MANOSEVITZ, $M$. Early experience, genotype, and temperament in Mus musculus. Journal of Comparative \& Physiological Psychology. 1963, 56. $622-629$.

ROBERTS, L. E. Central, peripheral, and artifactual determinants of skin resistance in the mouse. Journal of Comparative \& Physiological Psychology. $1967 \mathrm{a}, 64,318-328$.

ROBERTS, L. E. Changes in skin conductance and operant behavior during discriminative CER conditioning in the mouse. Paper presented at the annual meeting of the Eastern Psychological Association, Boston, April 1967b.

WOLL, $S$. V. Conditioned-suppression in the rat: Concomitant changes in skin conductance and sikin potential as measured by an improved grid technique. Unpublished Master's thesis, McMaster University, 1968.

\section{NOTE}

1. Roberts, L. E., \& Young, R. Heart rate is related to movement during classical conditioning in the rat, but skin conductance and potential are not. Submitted for publication.

\section{The effects of a tranquilizer on the immobility reaction in chickens: Additional support for the fear hypothesis}

GORDON G. GALLUP, JR., RICHARD F. NASH, and CHARLES W. BROWN Tulane University, New Orleans, La. 70118

In terms of number of inductions needed to elicit the immobility response, chickens given metoserpate HCL (Pacitran) were found to be significantly less susceptible to immobility than controls. The duration of resulting immobility reactions was also found to be inversely related to drug dosage levels, with controls remaining immobile over three times longer than chicks receiving an optimal dosage. The results were interpreted as lending support to the notion that fear is what underlies tonic immobility reactions in young chicks.

Recent research has provided considerable support for the idea that the tonic immobility response in chickens represents a fear reaction. The application of standard fear-induction procedures such as electric shock (Gallup, Creekmore, \& Hill, 1970) and loud noise (Gallup, Nash, Potter, \& Donegan, 1970), as well as confrontation with potential predators (Gallup \& Nash, 1970), have all been shown to reliably enhance immobility reactions in young chicks.

An alternate approach to testing the fear hypothesis would be to employ procedures designed to reduce, rather than increase, fear. It is well known, for example, that handling, familiarization, and repeated testing cause the immobility response in chickens to wane (Gilman, Marcuse, \& Moore, 1950; Ratner \& Thompson, 1960). Another possibility might be the use of tranquilizing agents as a technique for reducing fear or arousal. Although numerous attempts to look at the effect of various drugs on the immobility reaction have been made, the results are contradictory (see review by Ratner, 1967).

Part of the problem resides in the fact that there are sometimes large species differences in response to a given tranquilizing agent (e.g., Garren \& Hill, 1957). Another difficulty relates to the technique employed in administering the drug. Pilot data from our laboratory indicate that injections (IV or IM) may, by virtue of their inherent fear and/or stress-producing effects, mask the effect of the drug on immobility; e.g., chickens given isotonic saline or chlorpromazine injections have often remained immobile for over $2 \mathrm{~h}$.

Recently, a new water-soluble tranquilizer (Pacitran, CIBA) was developed specifically for use with poultry, and numerous reports (e.g., Belloff \& Hsu, 1963; Champion, Zindel, Ringer, \& Wolford, 1966) have documented its effectiveness for reducing emotionality in chickens subject to commercial handling. Moreover, the drug can be administered by simply adding it to drinking water following a specified 